\title{
Microscopic polyangiitis: from pathogenesis to treatment
}

\begin{abstract}
Microscopic Polyangiitis (MPA) is a systemic pauci-immune necrotizing vasculitis of small-calibre vessels characterized by the absence of granulomas. MPA is an autoimmune disease but its aetiology remains obscure. MPA is associated with presence of antineutrophil cytoplasmatic autoantibodies (ANCAs) but not all patients with MPA have ANCAs. There is strong evidence that not all ANCAs are pathogenetic. It seems that specific epitopes determine ANCAs pathogenicity. Given that MPA is a systemic vasculitis, multiple organs could be affected resulting in a wide spectrum of signs and symptoms. The kidneys and lungs are the most typical organs involved in MPA. Notably, MPA is the major cause of pulmo-renal syndrome. MPA has a poor prognosis if not treated but the use of aggressive immunosuppressive treatment has improved the prognosis and the patient's survival. Rituximab could be considered as an alternative treatment for severe disease, for patients who do not respond adequately to the immunosuppressive treatment and for patients with relapses. However, the long-term safety of Rituximab in MPA is unknown and it should be elucidated by further studies. Interestingly, kidney transplantation is safe and effective and it has a good prognosis in MPA patients with ESRD.
\end{abstract}

Keywords: ANCAs, microscopic polyangiitis, treatment, vasculitis
Volume 5 Issue 2 - 2017

Periklis Dousdampanis,' Stelios F

Assimakopoulos, ${ }^{2}$ Konstantina Trigka'

'Hemodialysis Unit Kyanos Stavros, Greece

${ }^{2}$ Department of Internal Medicine, University Hospital of Patras, Greece

Correspondence: Periklis Dousdampanis, Hemodialysis Unit Kyanos Stavros, Germanou II 5, Patras, 26225, Greece, Tel 302610243। I0, Fax 302610243I39,

Email dousdampanis@yahoo.gr

Received: June 26, 2017 | Published: August 17, 2017

\section{Introduction}

The term vasculitides refers to a group of inflammatory disorders involving any size or type of vessel. Microscopic Polyangiitis (MPA) is a systemic pauci-immune vasculitis of glomerular capillaries leading to necrotizing glomerulonephritis. ${ }^{1}$ Renal involvement is particularly frequent in small vessel systemic necrotizing vasculitis called anti-neutrophil cytoplamic autoantibody associated vasculitis (AAV), including MPA, granulomatosis with polyangiitis (GPA) and eosinophilic granulomatosis with polyangiitis. Renal involvement in MPA is of particular importance because it is associated with poor prognosis and increased mortality. ${ }^{2}$ In particular, the presence of renal impairment and dialysis dependence at diagnosis increases the risk of death in AAV patients. ${ }^{3,4}$

The main clinical presentation in MPA is rapidly progressive glomerulonephritis (RPGN) characterized by rapid decrease of glomerular filtration rate (GFR), microscopic haematuria, erythrocyte cast ,presence of proteinuria (usually less than $3 \mathrm{~g}$ ) and hypertension. Lungs are usually also affected in MPA. Lung manifestations consist of diffuse alveolar haemorrhage due to pulmonary capillaritis. ${ }^{1,2}$ The pathogenesis of MPA is unknown. There is increasing evidence that environment factors in association with a genetic predisposition are involved in the pathogenesis of MPA. In addition, a pathogenic role for ANCAs has been proposed..$^{5}$ Histological confirmation of vasculitis remains still the gold standard of the diagnosis of MPA. The therapy of MPA consists of remission induction strategies in order to achieve remission of the disease and remission maintenance strategies. Kidney transplantation should be considered as first option in AAV patients which are in remission more than one year. ${ }^{6}$

\section{History}

In 1866, Kaussmal and Maier were the first to describe completely a case report of a 27-year old man with systemic vasculitis. The term polyarterites nodosa $(\mathrm{PN})$ was introduced to describe all the patients with noninfectious arteritis. ${ }^{1}$ In 1923, a new distinct entity from PN was described the so-called "microscopic form of periarteritis nodosa" characterized both by the presence of glomerulonephritis and non-granulomatous inflammation. ${ }^{7}$ Later, a stretch correlation of "microscopic form of periarteritis nodosa" with Wegener's granulomatosis and Churg-Strauss syndrome now called granulomatosis with polyangiitis (GPA) and eosinophilic granulomatosis with polyangiitis was described respectively. ${ }^{8}$ In 1985 , the term microscopic polyarteritis was replaced by the term microscopic polyangiitis. ${ }^{9}$ After three years Jennete and Falk reported an association of the disease with ANCAs. ${ }^{10}$ In, 1994 the Chapel Hill Consensus Conference (CHCC) introduced the term microscopic polyangiitis describing a small vessel vasculitis characterized by rapid progressive glomerulonephritis and pulmonary capilaritis with the absence of immune complex deposition on immunofluorescence. ${ }^{2}$ Finally, according to 2012 revision of CHCC classification, MPA was reported as a pauci -immune small-vessel vasculitis with absence of granulomas or eosinophilia associated with myeloperoxidase (MPOANCA). ${ }^{11,12}$

\section{Epidemiology}

An increased incidence has been reported especially in southern Europe. ${ }^{13}$ The incidence of MPA is 2,7 to 94 per 1 million with a slight male predominance. ${ }^{14}$ However, other data do not correlate the incidence of MPA with the latitude. ${ }^{1,15,16}$ The average age of onset is between 50 and 60 years. ${ }^{13,17,18}$

\section{Pathogenesis}

The aetiology of MPA is unknown. Environmental factors including silica exposure may be implicated in the pathogenesis but their exact role is obscure. ${ }^{19}$. There is ongoing evidence that MPA is an autoimmune disease in which ANCAs play a crucial role. In the majority of the cases MPO-ANCA has been detected but autoantibodies against Proteinase 3 (PR3) have also been reported in MPO patients. ${ }^{20}$ Data from animal studies, reported that ANCAs may activate (primed) neutrophils to produce reactive oxygen species and several 
lytic enzymes resulting in endothelial detachment and lysis. ${ }^{21-23}$ In accordance, passage of MPO-ANCA from mother to fetus may cause pulmonary hemorrhage and renal failure to newborn. ${ }^{24}$ Interestingly, not all ANCAs are pathogenic. ${ }^{25}$ Paradoxically a subpopulation of patients which fulfill the criteria for ANCA associated vasculitis are negative for both MPO and PR3 -ANCAs. ${ }^{26,27}$ In this regard, there are no differences in kidney lesions between patients with ANCA negative pauci immune vasculitis and ANCA positive patients. ${ }^{25}$ Whereas, more severe renal lesions including glomerulosclerosis and interstitial fibrosis observed in ANCA negative and MPOANCA positive patients in comparison with PR3-ANCA patients. ${ }^{25}$ Interestingly, overall mortality was the same between ANCA-positive and ANCA negative patients with vasculitis. ${ }^{27}$. Additionally, it should be emphasized that some MPA patients are ANCA negative suggesting another pathogenetic mechanism different from the above. In support, rituximab is also effective in ANCA negative patients with vasculitis suggesting that there is an alternative pathogenic mechanism different from this mediated by the production of ANCA by the B cells. ${ }^{28}$

There is evidence that for the development of MP a specific type of ANCA against specific epitopes is required. ${ }^{25}$ Roth et al. ${ }^{29}$ reported a total of 25 different epitopes of MPO-ANCA in patients with active disease, with remissions, ANCA-negative vasculitis and controls. Interestingly,12 of these epitopes were correlated to active disease, while 8 epitopes were also identified in healthy subjects and for this reason were termed nature epitopes. Interestingly, there is an association of ANCAs with different MHC class II genes suggesting a genetic predisposition. ${ }^{30}$

\section{Clinical manifestations}

MPA is a systemic vasculitis indicating that several organs can be affected. Renal involvement occurs in the majority of the cases and it is manifested by microscopic haematuria, mild proteinuria, casts and deterioration of renal function due to rapidly progressive glomerulonephritis. ${ }^{31}$ Notably, MPA may be restricted only to the kidney without other organ involvement. This rare condition is characterized as idiopatic necrotizing, crescentic glomerulonephritis. Pulmonary involvement occurs less frequently. MPA patients with lung involvement present dyspnea, cough and hemoptysis due to alveolar haemorrhage. Capillaritis with fibrinoid necrosis is the typical pathologic feature in MPO patients with lung involvement. ${ }^{31}$ Of note, MPA is the most frequent cause of pulmonary-renal syndrome. Besides the general malaise and myalgias with arhralgias, skin involvement including purpura, livedo reticularis, nodules, urticaria and skin ulcers may be the initial presenting signs. ${ }^{31}$ Gastrointestinal manifestations consist of abdominal pain and bleeding. Neurological involvement is common. ${ }^{30}$ Peripheral neuropathy (mononeuritis-symmetrical polyneuropathy) is the predominant manifestation while involvement of the central nervous system has also been reported. ${ }^{31}$ Ear nose and throat manifestations are less frequent in MPA patients. ${ }^{31}$

\section{Diagnosis}

At present, there is no specific diagnostic tool for MPA. Diagnosis should be based on clinical symptoms and signs from various systems affected and on pathological feature. Nonspecific markers of inflammation including leucocytosis, increased sedimentation velocity, increased PCR and normocitic anaemia are indicative but not diagnostic for MPA disease. The absence of ANCAs does not exclude the diagnosis. Pathological feature of pauci-immune necrotizing small-vessel vasculitis in biopsy, confirms the diagnosis of MPA. The absence of granulomatous inflammation differentiates MPA from GPA. $^{32}$

\section{Prognosis}

The prognosis of MPA is poor without treatment (annual mortality rate above 90\%). Interestingly, the introduction of aggressive immunosuppressive drugs has substantially improved the prognosis. ${ }^{2}$ More specifically, the cumulative survival of MPA patients with renal involvement at 1 and 5 years was $82 \%$ and $76 \%$ respectively. ${ }^{5}$ ESRD observed in $28 \%$ of MPA patients. Risk factors dor ESRD were serum creatinine at the time of the diagnosis, African American race and severity of histological lesions at biopsy. ${ }^{5}$ The mortality in ESRD patients due to MPA was around $50 \%$ the prognosis was worse in patients with pulmo-renal syndrome. ${ }^{33,34}$ Death occurring during the first year of MPA immunosuppressive treatment was related primary to insufficient treatment response, several infections, cardiovascular disease and malignancy. ${ }^{4}$. Disease relapse occurred in $35 \%$ of MPA patients. ${ }^{5}$

\section{Treatment}

Induction treatment consists of immunosuppressant's including cyclophosphamide ( $2 \mathrm{mg} / \mathrm{kgr} /$ day for 3-6 months) plus corticosteroids $(1 \mathrm{mg} / \mathrm{kgr} /$ day $)$ with tapering reviewed in. ${ }^{31}$ In more severe cases can precede the use of methylprednisolone iv (500mg or 1 gr 3 times). Intravenous pulse cyclophosphamide $(15 \mathrm{mg} / \mathrm{kgr})$ is also effective compared to oral treatment with lesser side effects. In more severe cases, with renal failure and lung involvement plasma exchange is indicated (seven exchanges over 2 weeks) reviewed in. ${ }^{31}$ Use of plasma infusion is limited to patients with relapse or to those resistant to immunosuppression treatment. ${ }^{7}$ The long term effect of plasmapheresis on patients' outcome is unknown. Whether duration of plasma exchange therapy should be tailored to ANCA titers has not been yet studied.

Once remission (absence of clinical manifestations of GN) has been obtained, maintenance therapy with Azathioprine (AZA) (2mg/ $\mathrm{kgr} / \mathrm{day}$ ) for 12 months should be added. Mycophenolate mofetil (MMF) is an alternative but it is less effective than AZA reviewed in. ${ }^{31}$ Rituximab (anti-CD20) $(375 \mathrm{mg} / \mathrm{m} 2$ once weekly for 4 weeks) plus corticosteroids per os even without maintenance treatment has been proposed as an alternative to immunosuppressants (cyclophosphamide switched to AZA as maintenance therapy) for induction of remission in severe cases with renal involvement and in patients which do not respond to immunosuppression reviewed in. ${ }^{31}$ Moreover, Rituximab could be considered as another effective option for the relapses. However, the long-term toxicity of Rituximab should be elucidated by further studies.

\section{Renal transplantation}

Despite the major advances in the treatment of MPA, a large number of patients with MPA develop end stage renal disease (ESRD). ${ }^{35}$ AAV patients with ESRD on dialysis have a worse prognosis compared to those without dialysis independent renal function. ${ }^{36}$ However, the overall mortality of AAV patients on dialysis is similar to those non-diabetic patients on dialysis. ${ }^{37-39}$ Renal transplantation (RTx) is the first choice of treatment in these patients. According to Kidney Disease: Improving Global Outcomes (KDIGO) Guidelines for Glomerulonephritis. ${ }^{6} \mathrm{RTx}$ is another therapeutic option in AAV patients with more than one year of disease remission. ${ }^{6}$ Of note, the definition of the remission is not widely acceptable by all but usually it requires the absence of systemic clinical symptoms. In addition, ANCA positivity at the time of RTx should not be considered as a contraindication. ${ }^{35}$ The risk of relapse is present but usually it decreases after RTx due to the more efficient anti-rejection therapy 
and the use of the immunosuppressant's. ${ }^{40,41}$ The treatment for disease relapses after transplantation in AAV patients does not differ from that of non kidney transplanted AAV patients raging the increase dose of corticosteroids to plasma exchange therapy based on the severity of the disease. ${ }^{41,42}$ Notably the use of cyclophosphamide remains also the gold standard and it has been successfully used in disease relapses after RTx. Rituximab could be considered as an alternative option to treat relapses after kidney transplantation. ${ }^{43,44}$

\section{Conclusion}

i. Microscopic polyangiitis is a systemic idiopathic autoimmune disease involving small-calibre blood vessels.

ii. ANCAs under certain circumstances are pathogenic.

iii. Multiple organs are affected but predominantly kidneys and lungs are involved. Kidney involvement is characterized by rapidly progressive glomeulonephritis whereas diffuse alveolar haemorrhage due to capillaritis is the main pulmonary manifestation.

iv. The diagnosis is based on clinical symptoms and ANCAs detection but tissue biopsy is the gold standard.

v. Older age, females, serum creatinine at diagnosis, chronic lesions and crescents at renal biopsy and response to therapy and flares are predictive factors for kidney function.

vi. The choice of therapy should be based on disease severity. Treatment strategies include cyclophosphamide with corticosteroids as therapy induction and Azathioprine as a remission maintenance therapy. However, Rituximab should be considered as an alternative therapy for patients who do not respond to immunosuppressant's and for disease relapses.

vii. Kidney transplantation is safe and effective and has a good prognosis in AAV patients with ESRD.

\section{Acknowledgements}

None.

\section{Conflicts of interest}

All authors declared there are no conflicts of interest.

\section{Funding}

None.

\section{References}

1. Chung SA, Seo P. Microscopic polyangiitis. Rheum Dis Clin North Am. 2010;36(3):545-558.

2. Greco A, De Virgilio A, Rizzo MI, et al. Microscopic polyangiitis:Advances in diagnostic and therapeutic approaches. Autoimmun Rev. 2015;14:837-844.

3. Mukhtyar C, Flossmann O, Hellmich B, et al. Outcomes from studies of antineutrophil cytoplasm antibody associated vasculitis:a systematic review by the European League Against Rheumatism systemic vasculitis task force. Ann Rheum Dis. 2008;67(7):1004-1010.

4. Flossmann O, Berden A, de Groot K, et al. Long-term patient survival in ANCA-associated vasculitis. Ann Rheum Dis. 2011;70(3):488-494.

5. Kallenberg CG. The diagnosis and classification of microscopic polyangiitis. Autoimmun. 2014;48-49:90-93.
6. Kidney Disease:Improving Global Outcomes (KDIGO) Glomerulonephritis Working Group. KDIGO clinical practice guideline for glomerulonephritis. Kidney Int. 2012;Suppl 2:139-274.

7. Wohlwill F. Über die nur mikroskopisch erkennbare Form der Periarterites nodosa. Virchows Arch Pathol Physiol. 1923;246:36.

8. Goldman G, Churg J. Wegener's granulomatosis:pathology and review of the literature. AMA Arch Pathol. 1954;58(6):533-553.

9. Savage CO, Winearls CG, Evans DJ, et al. Microscopic polyarteritis: presentation, pathology and prognosis. QJ Med. 1985;56(220):467-483.

10. Falk RJ, Jennette JC. Anti-neutrophil cytoplasmic autoantibodies with specificity for myeloperoxidase in patients with systemic vasculitis and idiopathic necrotizing and crescentic glomerulonephritis. $N$ Engl $J$ Med. 1988;318(25):1651-1657.

11. Jennette JC, Falk RJ, Andrassy K, et al. Nomenclature of systemic vasculitides. Proposal of an international consensus conference. Arthritis Rheum. 1994;37(2):187-192.

12. Jennette JC, Falk RJ, Bacon PA, et al. 2012 revised International Chapel Hill Consensus Conference Nomenclature of Vasculitides. Arthritis Rheum. 2013;65(1):1-11.

13. Mahr A, Guillevin L, Poissonnet M, et al. Prevalences of polyarteritis nodosa, microscopic polyangiitis, Wegener's granulomatosis, and Churg-Strauss syndrome in a French urban multiethnic population in 2000:a capture-recapture estimate. Arthritis Rheum. 2004;51(1):92-99.

14. Koldingsnes W, Nossent H. Epidemiology of Wegener's granulomatosis in northern Norway. Arthritis Rheum. 2000;43(11):2481-2487.

15. Mohammad AJ, Jacobsson LT, Mahr AD, et al. Prevalence of Wegener's granulomatosis, microscopic polyangiitis, polyarteritis nodosa and Churg-Strauss syndrome within a defined population in southern Sweden. Rheumatology (Oxford). 2007;46(8):1329-1337.

16. Frankel SK, Jayne D. The pulmonary vasculitides. Clin Chest Med. 2007;31(3):519-536.

17. Guillevin L, Durand-Gasselin B, Cevallos R, et al. Microscopic polyangiitis: clinical and laboratory findings in eighty-five patients. Arthritis Rheum. 1999;42(3):421-130.

18. Agard C, Mouthon L, Mahr A, et al.Microscopic polyangiitis and polyarteritis nodosa:how and when do they start? Arthritis Rheum. 2003;49(5):709-715.

19. Hogan SL, Satterly KK, Dooley MA, et al. Silica exposure in antineutrophil cytoplasmic autoantibody-associated glomerulonephritis and lupus nephritis. J Am Soc Nephrol. 2001;12(1):134-142.

20. Flossmann O, Berden A, de Groot K, et al. Long-term patient survival in ANCA-associated vasculitis. Ann Rheum Dis. 2011;70(3):488-494.

21. Little MA, Smyth CL, Yadav R, et al. Antineutrophil cytoplasm antibodies directed against myeloperoxidase augment leukocytemicrovascular interactions in vivo. Blood. 2005;106(6):2050-2058.

22. Xiao H, Heeringa $\mathrm{P}, \mathrm{Hu} \mathrm{P}$, et al. Antineutrophil cytoplasmic autoantibodies specific for myeloperoxidase cause glomerulonephritis and vasculitis in mice. J Clin Invest. 2002;110(7):955-963.

23. Huugen D, Tervaert JW, Heeringa P. Antineutrophil cytoplasmic autoantibodies and pathophysiology:new insights from animal models. Curr Opin Rheumatol. 2004;16(1):4-8

24. Schlieben DJ, Korbet SM, Kimura RE, et al. Pulmonary-renal syndrome in a newborn with placental transmission of ANCAs. Am J Kidney Dis. 2005;45(4):758-761.

25. Land J, Rutgers A, Kallenberg CG. Anti-neutrophil cytoplasmic autoantibody pathogenicity revisited:pathogenic versus non-pathogenic anti-neutrophil cytoplasmic autoantibody. Nephrol Dial Transplant. 2014;29(4):739-745. 
26. Hedger N, Stevens J, Drey N, et al. Incidence and outcome of pauciimmune rapidly progressive glomerulonephritis in Wessex, UK:a 10year retrospective study. Nephrol Dial Transplant. 2000;15(10):15931599.

27. Eisenberger U, Fakhouri F, Vanhille P, et al. ANCA-negative pauciimmune renal vasculitis:histology and outcome. Nephrol Dial Transplant. 2005;20(7):1392-1399.

28. Khan A, Lawson CA, Quinn MA, et al. Successful Treatment of ANCANegative Wegener's Granulomatosis with Rituximab. Int J Rheumatol. 2010:84606.

29. Roth AJ, Ooi JD, Hess JJ, et al. Epitope specificity determines pathogenicity and detectability in ANCA-associated vasculitis. $J$ Clin Invest. 2013;123(4):1773-1783.

30. Lyons PA, Rayner TF, Trivedi S, et al. Genetically distinct subsets within ANCA-associated vasculitis. N Engl J Med. 2012;367:214-223.

31. Villiger PM, Guillevin L. Microscopic polyangiitis: Clinical presentation. Autoimmun Rev. 2010;9(12):812-819.

32. Watts R, Lane S, Hanslik T, et al. Development and validation of a consensus methodology for the classification of the ANCA-associated vasculitides and polyarteritis nodosa for epidemiological studies. Ann Rheum Dis. 2007;66(2):222-227.

33. Booth AD, Almond MK, Burns A, et al. Outcome of ANCA-associated renal vasculitis:a 5-year retrospective study. Am J Kidney Dis. 2003;41(4):776-784.

34. Niles JL, Böttinger EP, Saurina GR, et al. The syndrome of lung hemorrhage and nephritis is usually an ANCA-associated condition. Arch Intern Med. 1996;156(4):440-445.

35. Hruskova Z, Geetha D, Tesar V. Renal transplantation in anti-neutrophil cytoplasmic antibody-associated vasculitis. Nephrol Dial Transplant. 2015;30 Suppl 1:1159-163.
36. de Joode AA, Sanders JS, Stegeman CA. Renal survival in proteinase 3 and myeloperoxidase ANCA-associated systemic vasculitis. Clin J Am Soc Nephrol. 2013;8(10):1709-1717.

37. Weidanz F, Day CJ, Hewins P, et al. Recurrences and infections during continuous immunosuppressive therapy after beginning dialysis in ANCA-associated vasculitis. Am J Kidney Dis. 2007;50(1):36-46.

38. Tang W, Bose B, McDonald SP, et al. The outcomes of patients with ESRD and ANCA-associated vasculitis in Australia and New Zealand. Clin J Am Soc Nephrol. 2013;8(5):773-780.

39. Hruskova Z, Stel VS, Jayne D, et al. Characteristics and Outcomes of Granulomatosis With Polyangiitis (Wegener) and Microscopic Polyangiitis Requiring Renal Replacement Therapy:Results From the European Renal Association-European Dialysis and Transplant Association Registry. Am J Kidney Dis. 2015;66(4):613-620.

40. Allen A, Pusey C, Gaskin G. Outcome of renal replacement therapy in antineutrophil cytoplasmic antibody-associated systemic vasculitis. $J$ Am Soc Nephrol. 1998;9(7):1258-1263.

41. Geetha D, Seo P. Renal transplantation in the ANCA-associated vasculitides. Am J Transplant. 2007;7(12):2657-2662.

42. Nachman PH, Segelmark M, Westman K, et al. Recurrent ANCAassociated small vessel vasculitis after transplantation:A pooled analysis. Kidney Int. 1999;56(4):1544-1550.

43. Murakami C, Manoharan P, Carter-Monroe N, et al. Rituximab for remission induction in recurrent ANCA-associated glomerulonephritis postkidney transplant. Transpl Int. 2013;26(12):1225-1231.

44. Geetha D, Seo P, Specks U, et al. Successful induction of remission with rituximab for relapse of ANCA-associated vasculitis post-kidney transplant: report of two cases. Am J Transplant. 2007;7(12):2821-2825. 